\section{A89 CHROMATIN-ACTIVATED NEUTROPHILS REPRESENT A MAJOR SOURCE OF INTERFERON $\alpha$}

Dennis Lindau, ${ }^{1}$ Armin Rabsteyn, ${ }^{1}$ Ina Kötter, ${ }^{2}$ Annette Igney, ${ }^{2}$ Marie-Christophe Boissier, ${ }^{3}$ Hans-Georg Rammensee, ${ }^{1}$ Patrice Decker ${ }^{1,3}{ }^{1}$ Department of Immunology, Institute for Cell Biology, University of Tübingen, Tübingen, Germany; ${ }^{2}$ Internal Medicine II, University Hospital, Tübingen, Germany; ${ }^{3}$ EA4222, Li2P, University of Paris 13, Bobigny, France

10.1136/ard.2010.148973.7

Background and objectives Nucleosomes (DNA-histones complexes) represent major autoantigens present in the circulation of systemic lupus erythematosus (SLE) patients. Interferon $\alpha$ (IFN $\alpha$ ) plays an important role in lupus development. Although activated plasmacytoid dendritic cells (pDC) are believed to be the main producers of IFN $\alpha$ in SLE, pDC represent a minor cell population and only a few lupus stimuli have been reported. On the other hand, neutrophils represent $50 \%$ of total blood leucocytes. Moreover, granulocytes are activated in SLE, especially neutrophils triggered by nucleosomes. Toll-like receptor (TLR) 9 recognises certain forms of DNA but its role in SLE is still not elucidated. The authors therefore sought to determine the cellular source of IFN $\alpha$ and the natural lupus stimuli, focusing on nucleosomeinduced IFN $\alpha$ production by neutrophils and the impact of TLR9.

Materials and methods Chromatin (mono-nucleosomes) was purified from calf thymus. Peripheral blood mononuclear cells (PBMCs) and neutrophils were isolated from healthy individuals and SLE patients. Mouse neutrophils were purified from the bone marrow. All cell types were characterised by flow cytometry. Cells were activated with different stimuli and IFN $\alpha$ production was estimated by flow cytometry (intracellular staining). IFN $\alpha$ secretion was confirmed by ELISA. Polymorphonuclear leucocyte activation was verified by measuring $\mathrm{CD} 66 \mathrm{~b}$ or $\mathrm{CD} 11 \mathrm{~b}$ upregulation (flow cytometry) and interleukin 8 (IL-8) or macrophage inflammatory protein 2 secretion (ELISA).

Results Isolated neutrophils produce IFN $\alpha$ upon stimulation with chromatin. IFN $\alpha$ secretion by neutrophils was confirmed by ELISA and was observed with steady-state neutrophils, and not pro-inflammatory neutrophils, from both healthy donors and SLE patients. Neutrophil-derived IFN $\alpha$ was detected in response to free chromatin, and not chromatin-containing immune complexes, as well as TLR9 agonists but not TLR4 agonists. Nucleosome-induced IFN $\alpha$ production by neutrophils was associated with IL-8 secretion and CD66b upregulation. Neutrophil priming by granulocyte colony-stimulating factor is not required. Autologous PBMC and platelets sustain IFN $\alpha$ secretion by chromatin-activated neutrophils in co-cultures. pDC also produced IFN $\alpha$ upon activation with nucleosomes or TLR9 agonists but to a lower extent. Finally, chromatininduced IFN $\alpha$ secretion occurs independently of TLR9 since neutrophils isolated from both wild-type and TLR9-deficient mice were activated.

Conclusions Neutrophils represent a major source of IFNa. This is the first report showing both that steady-state neutrophils can secrete IFN $\alpha$ and identifying a natural lupus stimulus 
involved. A key event is thus the presence of increased concentrations of circulating nucleosomes, a situation met in SLE patients. Chromatin-activated neutrophils may secrete IFN $\alpha$ early during the lupus disease, before immune complexes are produced, bypassing the classical mechanism involving pDC. 